\title{
Matrix metalloproteinase 7, soluble Fas and Fas ligand serum levels for predicting docetaxel resistance and survival in castration-resistant prostate cancer
}

\author{
Tibor Szarvas*** (D), Sabina Sevcenco ${ }^{\dagger}$, Orsolya Módos*, Dávid Keresztes*, \\ Péter Nyirády*, Anita Csizmarik*, Robin Ristl ${ }^{\S}$, Martin Puhr", Michèle J. Hoffmann**, \\ Christian Niedworok ${ }^{\ddagger}$, Boris Hadaschik ${ }^{\ddagger}$ Agnieszka Maj-Hes ${ }^{\dagger}$, Shahrokh F. Shariat ${ }^{\dagger}$ and \\ Gero Kramer ${ }^{\dagger}$
}

*Department of Urology, Semmelweis University, Budapest, Hungary, 'Department of Urology, Vienna General Hospital, Medical University Vienna, Vienna, Austria, "Department of Urology, Faculty of Medicine, University Duisburg-Essen, Essen, Germany, ${ }^{\S}$ Centre for Medical Statistics, Informatics, and Intelligent Systems, Medical University of Vienna, Vienna, "Experimental Urology, Department of Urology, Medical University of Innsbruck, Innsbruck, Austria, and * *Department of Urology, Medical Faculty, Heinrich-Heine-University Düsseldorf, Düsseldorf, Germany

\section{Objective}

To assess the predictive value of pre-chemotherapy matrix metalloproteinase 7 (MMP-7), soluble Fas (sFas) and Fas ligand (FasL) serum levels, as well as their changes during therapy.

\section{Patients and Methods}

Serum levels of MMP-7, Fas and FasL were determined by ELISA in 96 patients with castration-resistant prostate cancer (CRPC): 21 docetaxel-resistant patients who received one single series and 75 docetaxel-sensitive patients who received repeated series of docetaxel. In addition to the 96 pretreatment serum samples, 987 sera collected during chemotherapy were also analysed.

\section{Results}

Higher pretreatment serum MMP-7, sFas and prostatespecific antigen (PSA) levels were significantly associated with both docetaxel resistance $(P=0.007, P=0.001, P<0.001$, respectively) and shorter cancer-specific survival $(P<0.001$, $P=0.041, P<0.001$, respectively). High MMP-7 level remained an independent predictor of both docetaxel resistance (hazard ratio [HR] 2.298, 95\% confidence interval [CI]: 1.354-3.899; $P=0.002)$ and poor cancer-specific survival (HR 2.11, 95\% CI: 1.36-3.30; $P=0.001$ ) in multivariable analyses. Greater increase in MMP-7 levels in the second treatment holiday and greater increase in PSA levels in the first and second treatment holidays were predictive of survival.

\section{Conclusions}

Pretreatment serum MMP-7 levels may help to select patients with CRPC who are likely to benefit from docetaxel chemotherapy. Furthermore, MMP-7 levels alone or in combination with PSA levels could be used for therapy monitoring. Correlative studies embedded in clinical trials are necessary to validate these biomarkers for clinical decisionmaking.

\section{Keywords}

MMP-7, Fas, serum, prognosis, docetaxel, \#pcsm, \#ProstateCancer

\section{Introduction}

Docetaxel is currently one of the standard first-line therapies for patients with castration-resistant prostate cancer (CRPC) [1-3]. While CRPC is generally a docetaxel-sensitive disease, there is a large variability in its response because of inherent or acquired docetaxel resistance. Patients who complete a first series of treatment without experiencing disease progression are considered docetaxel-sensitive, while those who experience disease progression are docetaxel-resistant. The recommended treatment is one single series of docetaxel containing 6 to 10 cycles in a 3-week schedule for both sensitive and resistant patients; however, docetaxel-sensitive patients might benefit from retreatment with further series of docetaxel as this can result in prolonged disease control $[4,5]$. Unfortunately, most docetaxel-sensitive patients will develop resistance to the chemotherapeutic drug during further treatment series. Thus, ideally docetaxel would be administered only to those patients 
who are sensitive and therapy would only be continued until the development of resistance. In the last few years, several effective drugs, such as abiraterone-acetate, enzalutamide, cabazitaxel and alpharadin, with different therapeutic mechanisms of action have been approved for the treatment of CRPC before or after docetaxel therapy [6]. These therapeutic methods provide reasonable options for patients who are resistant to docetaxel.

Our ability to predict docetaxel sensitivity is limited. It is therefore important to explore predictive factors for the selection of patients who are likely to benefit from first-line docetaxel treatment. As drug resistance is an evolutionary process, monitoring of changes rather than a single snapshot of biomarker levels might be able to reflect the inherent sensitivity to docetaxel therapy, helping with timely detection or prediction of docetaxel resistance, and potentially leading to add-on or changes of therapies.

Matrix metalloproteinase 7 (MMP-7) has been associated with chemotherapy resistance. In head and neck cancer, Ansell et al. [7] performed comparative genome-wide transcript profiling and identified and validated MMP-7 as a predictive marker for chemotherapy resistance [7]. Similarly, Liu et al. [8] demonstrated that high MMP-7 tissue expression was associated with poor response and survival in patients with non-small-cell lung cancer treated with cisplatin-based chemotherapy [8]. In recent years, a growing body of evidence has been collected to support the hypothesis that MMP-7 is causally involved in therapy resistance by allowing tumour cells to escape from chemotherapy-induced apoptosis [9-14]. Interaction between the death receptor Fas, and its ligand FasL, plays a central role in chemotherapyinduced cell death. The degrading and downregulating effects of MMP-7 on receptor Fas and FasL have been identified as a key mechanism responsible for MMP-7-related chemotherapy resistance. In addition, we have previously shown that high MMP-7 serum levels are independently associated with worse cancer-specific survival in localized prostate cancer $(\mathrm{PCa})$ [15].

These data and the unmet need for predictive markers in docetaxel-treated patients with CRPC led us to determine the pre-therapy levels of and changes in MMP-7, soluble Fas (sFas) and FasL during docetaxel treatment with the aim of assessing their predictive value.

\section{Materials and Methods}

\section{Patient Characteristics}

This study included 96 patients with CRPC who were treated with docetaxel at a single tertiary care centre between April 2003 and February 2010. Patients who completed the planned docetaxel treatment course with a good PSA response and without experiencing radiographic progression were considered docetaxel-sensitive and underwent retreatment with docetaxel. After the first retreatment, further retreatments were offered based on the same criteria. Our institutional ethics committee approved the study protocol. PSA response was defined, according to the Prostate Cancer Clinical Trials Working Group Criteria I, as a PSA decline from baseline of $\geq 50 \%$ during the first chemotherapy series [16]. Radiographic progression was defined according to the Response Evaluation Criteria in Solid Tumours (RECIST) [17].

Of the 96 patients with CRPC, 21 received one single series of docetaxel with five to eight cycles. These single-treatment patients were considered docetaxel-resistant. The remaining 75 patients were treated with at least two series of docetaxel. As these patients experienced a PSA decline and no radiographic progression during the first series, we considered them to be initially docetaxel-sensitive.

Criteria for inclusion were: availability of serum samples at first docetaxel treatment; no more than two missing serum samples during treatment follow-up; no second tumours at baseline; and no further lines of therapies after docetaxel.

\section{Sample Selection for Analysis}

As we aimed to assess the value of MMP-7, sFas and FasL as pre-therapy predictive and/or therapy-monitoring markers, baseline concentrations and changes during docetaxel therapy were analysed. Serum samples from the first, third, seventh, 21st, 42nd and last day of the first series were assessed. For the patients who received a second docetaxel series, serum samples were obtained on day 1 , day 42 and the last day of therapy. In any additional series, sera from the first and last day were analysed. In all cases, serum samples from the time of first radiographic progression on docetaxel treatment and treatment holiday were analysed. Finally, in all cases, the last available serum sample after therapy failure was assessed and considered as samples at time of disease progression. Overall, 1083 serum samples from 96 patients were analysed.

\section{ELISA Analysis}

Patients' MMP-7, sFas and FasL serum levels were quantified using Quantikine ELISA kits (DMP700, DFS00, DFL00, respectively; R\&D Systems, Wiesbaden, Germany) according to the manufacturer's instructions. Dilutions were twofold for MMP-7 and FasL, and 20-fold for sFas.

\section{Statistical Analysis}

For paired comparisons between groups, the non-parametric, two-sided Wilcoxon rank-sum test was applied. Survival analyses were carried out using Kaplan-Meier curves, logrank tests and univariable Cox proportional hazards 
regression analysis. For multivariable analysis, Cox regression models were used. Variables with an effect on survival in univariable analysis $(P \leq 0.05)$ were considered in the Cox proportional hazards regression models. In calculations where dichotomizations were required, the median values were used as thresholds. Pearson's correlation coefficient was used to assess the relationship between MMP-7, sFas and FasL serum concentrations. Finally, we calculated concordance indices for survival predictive models [18]. All statistical analyses were two-sided; $P$ values $\leq 0.05$ were taken to indicate statistical significance. All tests were carried out using the sPss software package (19.0; SPSS, Chicago, IL, USA).

\section{Results}

\section{Clinical Background}

Overall, 131 cycles of docetaxel were administered to 21 patients with primary docetaxel resistance and 1099 cycles were administered to 75 docetaxel-sensitive patients. The total number of rechallenges was 151 . The median durations of the first, second and third treatment holidays were 18, 16 and 15 weeks, respectively (Table 1 ). A total of 19 patients received vinorelbine as first-line therapy and 27 patients received docetaxel in combination with estramustine phosphate.

Of the 96 patients with CRPC, 47 had metastases, while 22 patients were treated after failure of androgen deprivation therapy with rising PSA level but without metastasis on

Table 1 Patients' characteristics.

\begin{tabular}{lc}
\hline Total number of patients & 96 \\
Median (range) age at baseline, years & $69(47-86)$ \\
ECOG PS at enrolment & 65 \\
0 & 22 \\
1 & 9 \\
2 & $83(2-1212)$ \\
PSA at baseline, ng/mL & 68 \\
Bone metastasis & 16 \\
Lymph node metastasis (>2 cm) & 6 \\
Soft tissue lesions (lung/liver) & 52 \\
Previous prostatectomy & \\
Docetaxel therapy & 77 \\
1st-line & 19 \\
2nd-line & \\
Pretreatment & 69 \\
No (docetaxel only) & 27 \\
Yes (EMP) & $2(1-10)$ \\
Median (range) number of docetaxel series & $11(5-44)$ \\
Median (range) number docetaxel cycles & 21 \\
Single (only one series) & 75 \\
Retreatment (at least two series) & 93 \\
Number of patients who died & 84 \\
PCa-specific deaths & $85(27-363)$ \\
Median (range) follow-up, weeks
\end{tabular}

ECOG PS, Eastern Cooperative Oncology Group performance status; EMP, estramustine phosphate; FasL, fas ligand; MMP-7, matrix metalloproteinase 7; PCa, prostate cancer; sFas, soluble Fas. Pretreated with EMP and/or vinorelbine.
CT. The main patient characteristics are provided in Table 1.

The main endpoints of the analysis were overall and PCaspecific survival. Of 96 patients, 93 had died at last follow-up, of whom 84 died from PCa. The median survival time was 39 weeks in the single-treatment, docetaxel-resistant group compared with 117 weeks in the retreated and initially docetaxel-sensitive group. Fifty-seven of 75 retreated patients had radiographic progression according to RECIST criteria on docetaxel chemotherapy (during treatment series) and 56 during treatment holiday (Table 1).

\section{Associations of Clinicopathological Variables and Therapy Type with Serum PSA, MMP-7, sFas and FasL Levels at Baseline}

The associations of baseline serum levels of MMP-7, sFas and FasL with clinicopathological variables are shown in Table 2. Patients' age, Eastern Cooperative Oncology Group performance status (ECOG PS), presence of lymph node or visceral metastasis and previously performed radical prostatectomy had no significant impact on PSA, MMP-7, sFas or FasL levels. Pretreatment with other first-line therapy or with estramustine phosphate was correlated with decreased MMP-7 serum levels. PSA levels were fourfold higher in patients with bone metastatic CRPC.

\section{Associations between Clinicopathological Variables, Baseline and Serum Marker Levels with Response to Docetaxel}

MMP-7, sFas and PSA levels were significantly higher in docetaxel-resistant (single-treatment) patients compared with those who were initially docetaxel-sensitive (retreatment patients; $P=0.007, P=0.010$ and $P<0.001$, respectively). In a multivariate logistic regression model for the prediction of response to docetaxel therapy we assessed: (i) ECOG PS; (ii) formerly performed radical prostatectomy; (iii) presence of metastasis at baseline; (iv) pretreatment PSA level; (v) sFas level; and (vi) MMP-7 level. This analysis revealed the presence of metastasis (odds ratio [OR] 2.445, CI: 1.2164.147; $P=0.010$ ), MMP-7 (OR 2.298, CI: 1.354-3.899; $P=$ 0.002 ) and PSA (OR 2.681, CI: 1.612-4.458; $P<0.001$ ) to be independently associated with treatment response.

\section{Associations between Patients' Survival and Serum Levels of PSA, MMP-7, sFas and FasL at Baseline}

Univariable Cox analysis found previously performed radical prostatectomy to be weakly associated with favourable overall survival $(P=0.049)$ but not with PCa-specific survival $(P=$ 0.19 ). Furthermore ECOG PS score $>0$, presence of any metastasis, bone metastasis or visceral metastasis as well as MMP-7, sFas and PSA levels above the median were 


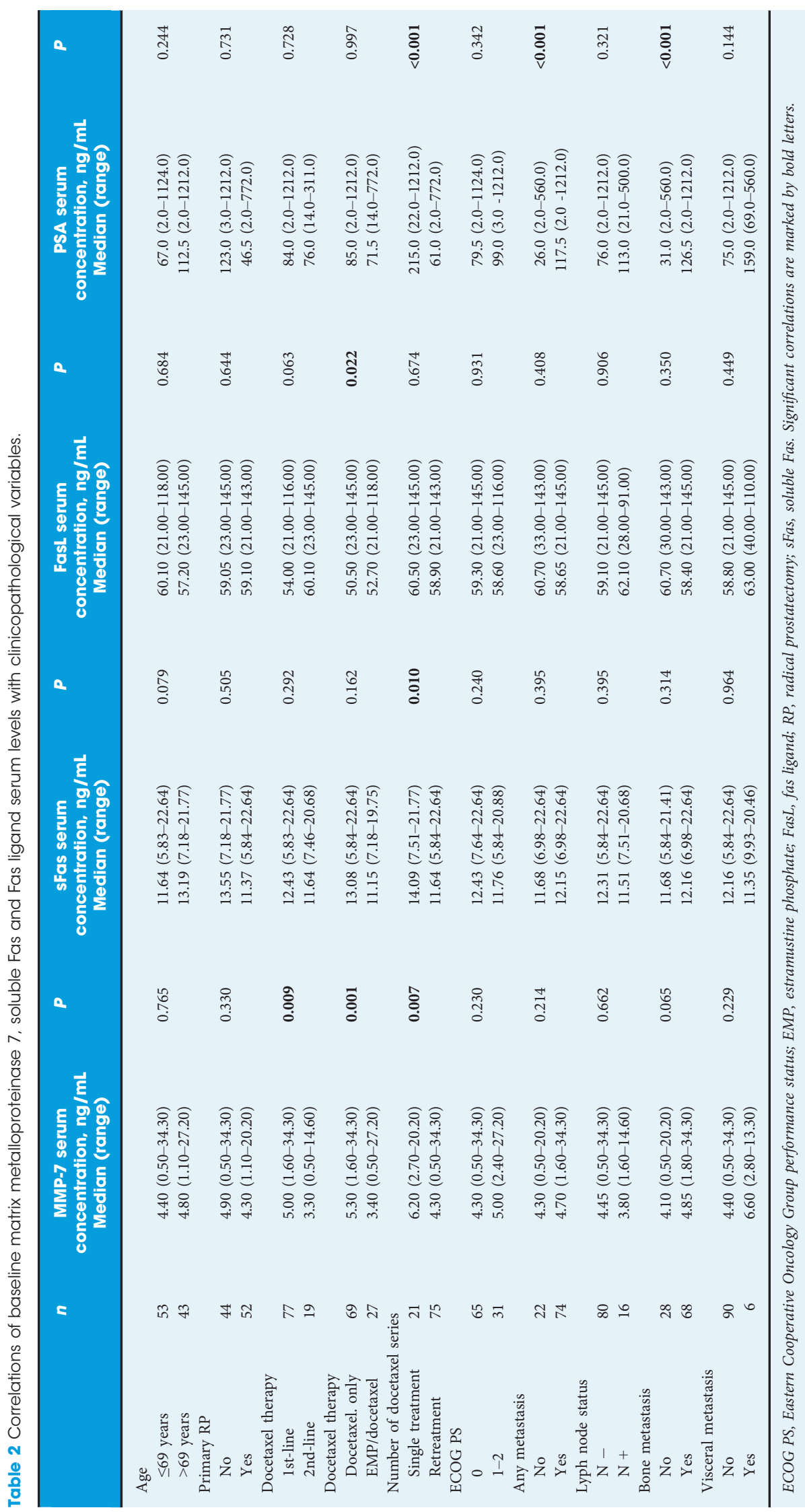


Table 3 Univariable Cox analysis.

\begin{tabular}{|c|c|c|c|c|c|c|}
\hline \multirow[t]{2}{*}{ Variables } & \multicolumn{3}{|c|}{ Overall survival } & \multicolumn{3}{|c|}{ Disease-specific survival } \\
\hline & HR & $95 \% \mathrm{CI}$ & $\boldsymbol{P}$ & HR & $95 \% \mathrm{CI}$ & $\boldsymbol{P}$ \\
\hline \multicolumn{7}{|l|}{ Age (years) } \\
\hline$\leq 65$ & Ref. & & & Ref. & & \\
\hline$>65$ & 1.466 & $0.916-2.348$ & 0.111 & 1.406 & $0.866-2.284$ & 0.168 \\
\hline \multicolumn{7}{|l|}{ Primary RP } \\
\hline No & Ref. & & & Ref. & & \\
\hline Yes & 0.658 & $0.434-0.998$ & 0.049 & 0.749 & $0.483-1.159$ & 0.194 \\
\hline \multicolumn{7}{|l|}{ Docetaxel therapy } \\
\hline 1st line & Ref. & & & Ref. & & \\
\hline 2nd line & 0.586 & $0.342-1.003$ & 0.051 & 0.607 & $0.348-1.057$ & 0.078 \\
\hline \multicolumn{7}{|l|}{ Docetaxel therapy } \\
\hline EMP/docetaxel & Ref. & & & Ref. & & \\
\hline Docetaxel only & 1.099 & $0.696-1.736$ & 0.685 & 0.995 & $0.626-1.584$ & 0.985 \\
\hline \multicolumn{7}{|l|}{ ECOG PS } \\
\hline 0 & Ref. & & & Ref. & & \\
\hline $1-2$ & 1.816 & $1.158-2.847$ & 0.009 & 1.841 & $1.154-2.935$ & 0.010 \\
\hline \multicolumn{7}{|l|}{ Any metastasis } \\
\hline No & Ref. & & & Ref. & & \\
\hline Yes & 2.791 & $1.631-4.774$ & $<0.001$ & 3.209 & $1.789-5.754$ & $<0.001$ \\
\hline \multicolumn{7}{|l|}{ Lymph node status } \\
\hline $\mathrm{N}-$ & Ref. & & & Ref. & & \\
\hline $\mathrm{N}+$ & 1.359 & $0.788-2.345$ & 0.270 & 1.400 & $0.796-2.462$ & 0.243 \\
\hline \multicolumn{7}{|l|}{ Bone metastasis } \\
\hline No & Ref. & & & Ref. & & \\
\hline Yes & 2.656 & $1.634-4.316$ & $<0.001$ & 2.849 & $1.702-4.768$ & $<0.001$ \\
\hline \multicolumn{7}{|l|}{ Visceral metastasis } \\
\hline No & Ref. & & & Ref. & & \\
\hline Yes & 3.331 & $1.431-7.754$ & 0.005 & 3.558 & $1.523-8.313$ & 0.003 \\
\hline \multicolumn{7}{|l|}{ MMP-7 serum concentration } \\
\hline \multirow[t]{2}{*}{$\leq 4.40 \mathrm{ng} / \mathrm{mL}$ (median) } & Ref. & & & Ref. & & \\
\hline & 2.268 & $1.466-3.508$ & $<0.001$ & 2.444 & $1.549-3.856$ & $<0.001$ \\
\hline \multicolumn{7}{|l|}{ sFas serum concentration } \\
\hline \multirow[t]{2}{*}{$<12.035 \mathrm{pg} / \mathrm{mL}$ (median) } & Ref. & & & Ref. & & \\
\hline & 1.690 & $1.111-2.571$ & 0.014 & 1.578 & $1.020-2.440$ & 0.041 \\
\hline \multicolumn{7}{|l|}{ FasL serum concentration } \\
\hline \multirow[t]{2}{*}{$<59.1$ pg/mL (median) } & Ref. & & & Ref. & & \\
\hline & 0.935 & $0.617-1.418$ & 0.751 & 0.917 & $0.594-1.415$ & 0.696 \\
\hline \multicolumn{7}{|l|}{ PSA serum concentration } \\
\hline \multirow[t]{2}{*}{$<83 \mathrm{ng} / \mathrm{mL}$ (median) } & Ref. & & & Ref. & & \\
\hline & 2.905 & $1.862-4.534$ & $<0.001$ & 3.145 & $1.975-5.008$ & $<0.001$ \\
\hline
\end{tabular}

ECOG PS, Eastern Cooperative Oncology Group performance status; EMP, estramustine phosphate; FasL, fas ligand; RP, radical prostatectomy; sFas, soluble Fas. Significant correlations are marked by bold letters.

significantly associated with both poor overall and diseasespecific survival (Table 3).

Multivariable analyses showed presence of any metastases, and high MMP-7 and PSA serum levels as independent and unfavourable prognostic factors for both overall and PCaspecific survival (Table 4). MMP-7 and PSA levels remained significant risk factors when included as continuous variables in the multivariable models (MMP-7: hazard ratio [HR] 1.067, 95\% CI: $1.024-1.113, P=0.002$; PSA: HR $1.002,95 \%$ CI: $1.001-1.003, P<0.001)$. In addition, because pretreatment with other chemotherapeutic agents and presence of metastasis were observed to influence MMP-7 baseline levels, we performed multivariable analysis excluding patients without metastasis who were pretreated with other chemotherapeutic agents $(n=49)$. Also in this model, high MMP-7 baseline level remained a significant and independent risk factor for patients' overall (HR 2.417, CI: 1.179-4.958; $P$ $=0.016$ ) and disease-specific survival (HR 2.774, CI: 1.2826.005; $P=0.010)$. The concordance index for the base model (ECOG PS score $>0$, presence of any metastasis, PSA $>83 \mathrm{ng} / \mathrm{mL}$ ) was 0.708 , which increased to 0.731 when MMP-7 ( $>4.4 \mathrm{ng} / \mathrm{mL})$ was added.

To show the independent prognostic value of PSA, MMP-7 and the metastatic status (Fig. $1 \mathrm{~A}-\mathrm{C}$ ), we combined these three prognostic factors for the prediction of patients' overall survival in Kaplan-Meier analysis (Fig. 1D-F) By combining PSA, MMP-7 and metastatic status, patients with low PSA and MMP-7 levels as well as absence of metastatic lesions had a survival probability of $100 \%$ after 150 weeks in contrast to the high-risk group (high PSA, high MMP-7 levels and metastatic lesions) in which only $\sim 5 \%$ of patients survived 150 weeks (Fig. 1F). Also in the subgroups of patients with 
Table 4 Multivariable Cox analysis.

\begin{tabular}{|c|c|c|c|c|c|c|}
\hline \multirow[t]{2}{*}{ Variables } & \multicolumn{3}{|c|}{ Overall survival } & \multicolumn{3}{|c|}{ Disease-specific survival } \\
\hline & HR & $95 \% \mathrm{CI}$ & $\boldsymbol{P}$ & HR & $95 \% \mathrm{CI}$ & $\boldsymbol{P}$ \\
\hline \multicolumn{7}{|l|}{ ECOG PS } \\
\hline 0 & Ref. & & & Ref. & & \\
\hline $1-2$ & 1.630 & $1.023-2.598$ & 0.040 & 1.669 & $1.028-2.710$ & 0.038 \\
\hline \multicolumn{7}{|l|}{ Any metastasis } \\
\hline No & Ref. & & & Ref. & & \\
\hline Yes & 2.087 & $1.180-3.692$ & 0.011 & 2.178 & $1.189-3.990$ & 0.012 \\
\hline \multicolumn{7}{|c|}{ MMP-7 serum concentration } \\
\hline$\leq 4.40 \mathrm{ng} / \mathrm{mL}$ & Ref. & & & Ref. & & \\
\hline$>4.40 \mathrm{ng} / \mathrm{mL}$ & 2.114 & $1.356-3.296$ & 0.001 & 2.134 & $1.372-3.476$ & 0.001 \\
\hline \multicolumn{7}{|c|}{ PSA serum concentration } \\
\hline$\leq 83 \mathrm{ng} / \mathrm{mL}$ & Ref. & & & Ref. & & \\
\hline$>83 \mathrm{ng} / \mathrm{mL}$ & 2.384 & $1.512-3.759$ & $<0.001$ & 2.270 & $1.621-4.216$ & $<0.001$ \\
\hline
\end{tabular}

ECOG PS, Eastern Cooperative Oncology Group performance status; HR, hazard ratio; MMP-7, matrix metalloproteinase 7. Significant correlations are marked by bold letters.

Fig. 1 Kaplan-Meier curves of overall survival according to (A) matrix metalloproteinase 7 (MMP-7) and (B) PSA levels and (C) presence of any metastasis (mets). The risk-stratification improves when MMP-7 (D) or metastatic status (E) are combined with PSA. The combination of three prognostic factors (MMP-7, PSA and the presence of any metastasis) results the most accurate risk assessment (F).

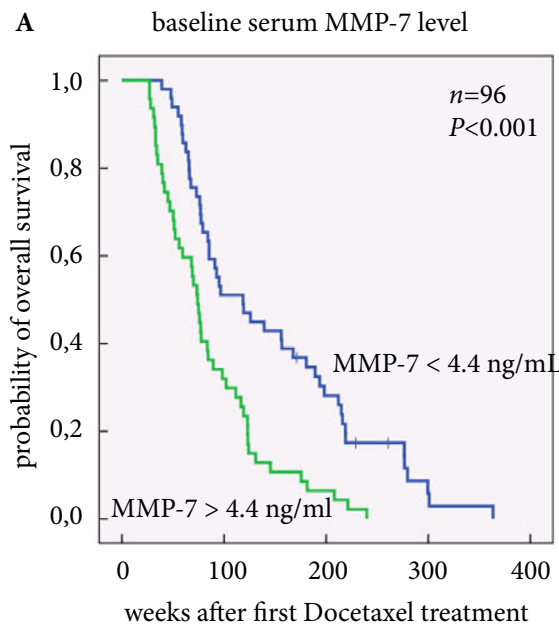

D combination PSA / MMP-7 levels

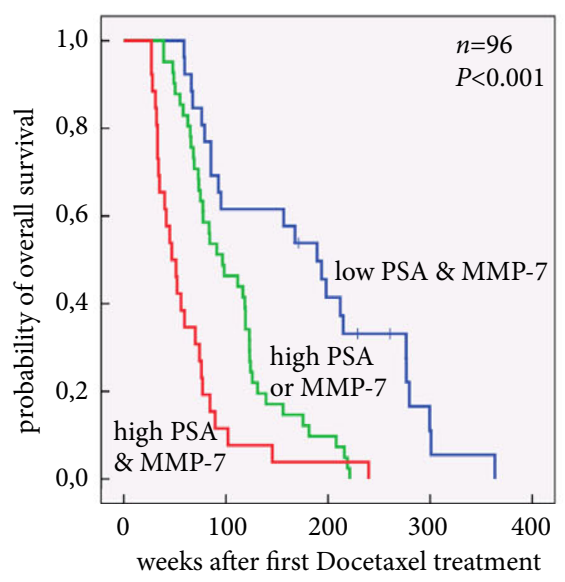

B baseline PSA level

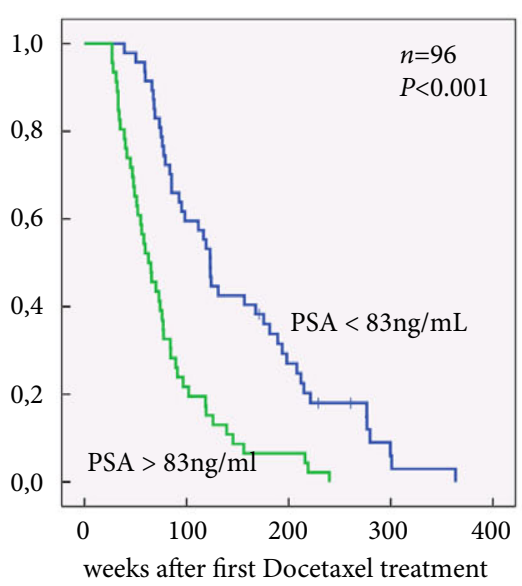

E combination PSA / presence of any mets.

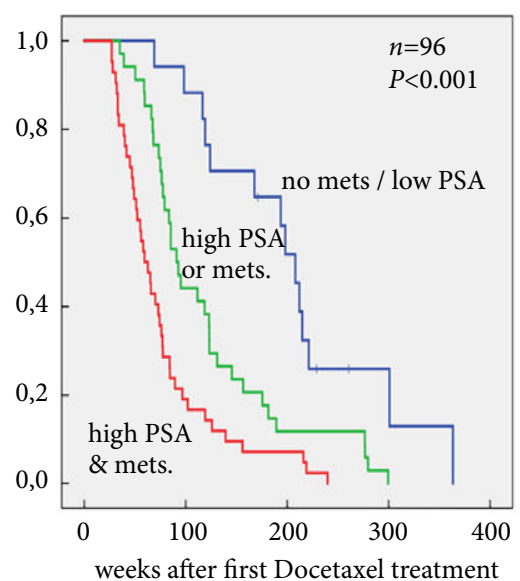

C

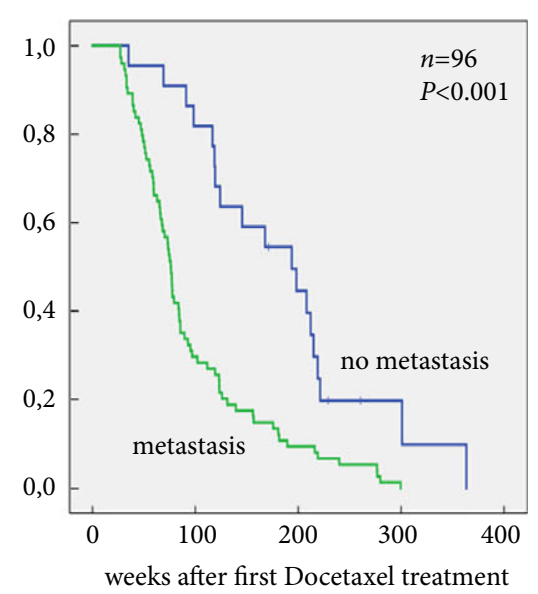

F

combination PSA / MMP-7 / presence of any

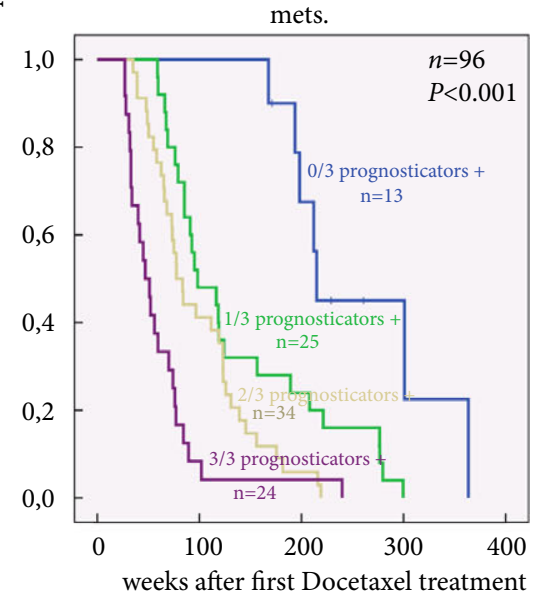


high PSA level and metastatic disease and with low PSA level without metastasis, high MMP-7 levels were significantly associated with shorter patient survival time (Fig. S1).

Compared with baseline levels, MMP-7, sFas and FasL levels were not significantly higher at progression (Table 1). In contrast, MMP-7 and sFas levels were significantly elevated after docetaxel failure and in the last samples before patients' deaths. PSA levels were mildly but not significantly elevated at the time of radiographic progression during treatment holiday $(P=0.093)$, but were significantly higher at PCa progression on docetaxel treatment (median 69 vs $129 \mathrm{ng} /$ $\mathrm{mL} ; P=0.027)$. Finally, PSA levels were fourfold elevated after docetaxel failure in comparison with baseline levels (median $440 \mathrm{ng} / \mathrm{mL}$ vs $123 \mathrm{ng} / \mathrm{mL} ; P<0.001$ ).

\section{Changes in MMP-7, sFas and FasL levels in response to docetaxel treatment}

PSA levels decreased during treatment series and increased in treatment holidays. MMP-7 levels showed no significant changes during docetaxel treatment if all patients were considered (Fig. 2). The subgroup analysis including only patients with high baseline MMP-7 levels $(>8 \mathrm{ng} / \mathrm{mL})$, however, revealed decreasing MMP-7 levels during treatment and increasing MMP-7 levels in treatment holidays (Fig. 2). In contrast, sFas and FasL levels exhibited no significant variations between different stages of docetaxel treatment.

\section{Predictive Value of Changes in MMP-7, sFas and FasL Serum Levels during Docetaxel Therapy}

Changes in PSA, MMP-7, sFas and FasL levels during treatment series were not predictive for overall or PCaspecific survival. In contrast, PSA increase in the first and second treatment holidays as well as MMP-7 increase in the second holiday proved to be predictive for both overall and PCa-specific survival independently of whether PSA and MMP-7 levels were calculated as continuous or categorized (median of change as cut-off) variables (Fig. S2). PSA and MMP-7 levels characteristically increased before or at the time of radiographic progression (Table S1 and Fig. S3).

\section{Correlations between MMP-7, sFas, FasL and PSA Levels}

Pearson's correlation analyses between marker levels showed a significant and linear correlation between sFas and MMP-7 serum concentrations $(r=0.437, P<0.001)$, and between MMP-7 and PSA levels $(r=0.217, P=0.037)$.

\section{Discussion}

In the present study, we determined MMP-7, sFas, FasL and PSA concentrations in over 1000 serum samples collected before and during docetaxel treatment from 96 patients with CRPC. Marker concentrations at baseline as well as their changes during docetaxel treatment were assessed for their predictive value for therapy effectiveness value. High pretreatment MMP-7, sFas and PSA levels were significantly associated with the presence of docetaxel resistance.

Furthermore, MMP-7 and PSA baseline levels proved to be independent and unfavourable predictive factors for survival in patients with CRPC treated with docetaxel chemotherapy. Changes in MMP-7 and PSA levels during treatment holidays were able to predict patients' prognosis.

MMP-7 is a member of the matrix metalloproteinase family capable of degrading extracellular matrix components and thereby promoting metastasis formation. In addition, MMP-7, based on its large substrate specificity, is involved in the regulation of several tumour-supporting cellular processes such as angiogenesis, apoptosis and epithelial-to-mesenchymal transition [19]. In PCa, MMP-7 has been shown to be focally expressed by the tumour cells [20]. A genome-wide screening study analysing $>200000$ single nucleotide polymorphism loci in 25000 patients with PCa and controls identified a region within the MMP-7 promoter that was associated with PCa risk [21]. In addition, Lynch et al. [22], using a rat PCa metastasis model, analysed the gene expression profiles of PCa cells from the centre of the metastasis compared with cells originated from the tumour-bone interface. They found MMP-7 to be one of the most significantly upregulated genes at the tumour-bone interface, suggesting its involvement in $\mathrm{PCa}$ bone metastasis formation. The causal involvement of MMP-7 in bone metastasis formation was confirmed using an in vivo MMP-7-deficient mouse model [22]. In accordance with that study, we recently found elevated serum MMP-7 levels in serum samples of PCa patients harbouring bone metastases compared with those without bone metastatic lesions [15]. These data together strongly suggest the causal involvement of MMP-7 in both formation and progression of PCa, and make MMP-7 a promising marker for monitoring tumour burden.

In addition, independent studies in different cancers including breast, lung and colon cancer confirmed the causal involvement of MMP-7 in the formation of chemotherapy resistance [9-14]. Elevated MMP-7 levels and decreased Fas expression was found in oxaliplatin-resistant colon cancer cells as compared with parental, oxaliplatin-sensitive cells. Sensitivity to chemotherapy could be restored by inhibition of MMP-7 which was accompanied by enhanced Fas expression, clearly indicating the causal involvement of MMP-7 in platinum resistance [11]. Similarly, in Ewing's sarcoma cells, MMP-7 was shown to proteolytically degrade FasL, resulting in decreased doxorubicin sensitivity [9]. MMP-7-expressing breast cancer cells exhibited reduced sensitivity to mitomycininduced apoptosis, and this effect was related to lower FasL levels in MMP-7 transgenic mice [14]. In lung 
Fig. 2 Changes in PSA, matrix metalloproteinase 7 (MMP-7), soluble Fas (sFas) and Fas ligand (FasL) serum levels during docetaxel treatment and treatment holidays. Red squares represent treatment days when serum samples were analysed. Curves were drawn by using the median values of marker concentrations. In case of MMP-7 a separate curve was drawn including only those patients with high MMP-7 serum levels ( $>8 \mathrm{ng} / \mathrm{mL}$ ) at baseline (dashed line).

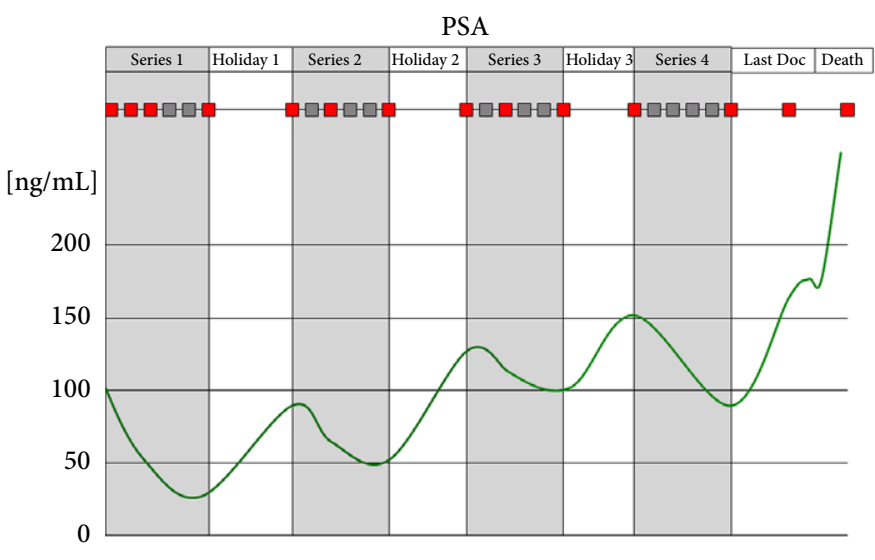

MMP-7

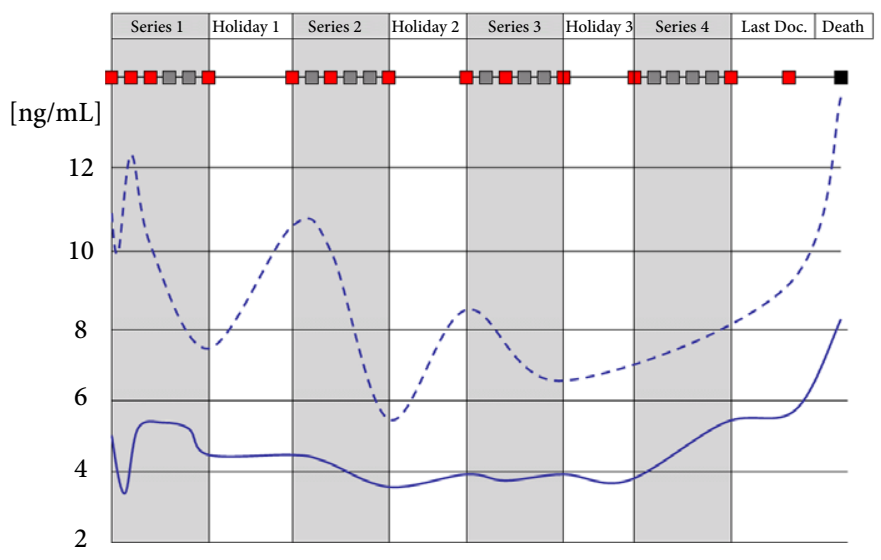

adenocarcinoma cells, exogenous exposure to MMP-7 significantly enhanced cisplatin resistance [13]. In accordance with this, MMP-7 immunostaining was predictive of therapy response and survival in patients with lung cancer treated with platinum-based chemotherapy [8]. These data suggest that MMP-7 is causally involved in apoptosis inhibition, enabling tumour cells to escape chemotherapy. Our results show that pretreatment MMP-7, sFas and PSA serum levels are higher in patients who are resistant to docetaxel. Accordingly, high pretreatment MMP-7 and PSA levels proved to be independent risk factors for survival in patients with CRPC treated with docetaxel chemotherapy. Based on these data, MMP-7 in combination with selected clinicopathological variables and PSA level might help to select patients for effective docetaxel treatment. Docetaxelsensitive patients might benefit from repeated docetaxel treatments until the development of docetaxel insensitivity. Conversely, men with docetaxel-resistant tumours might be spared from ineffective therapy and unnecessary toxicity. In

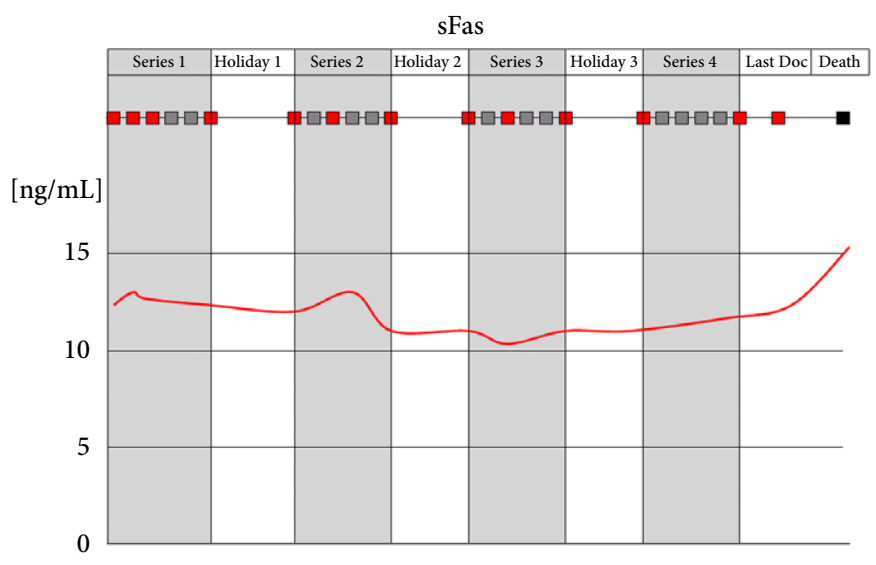

FasL

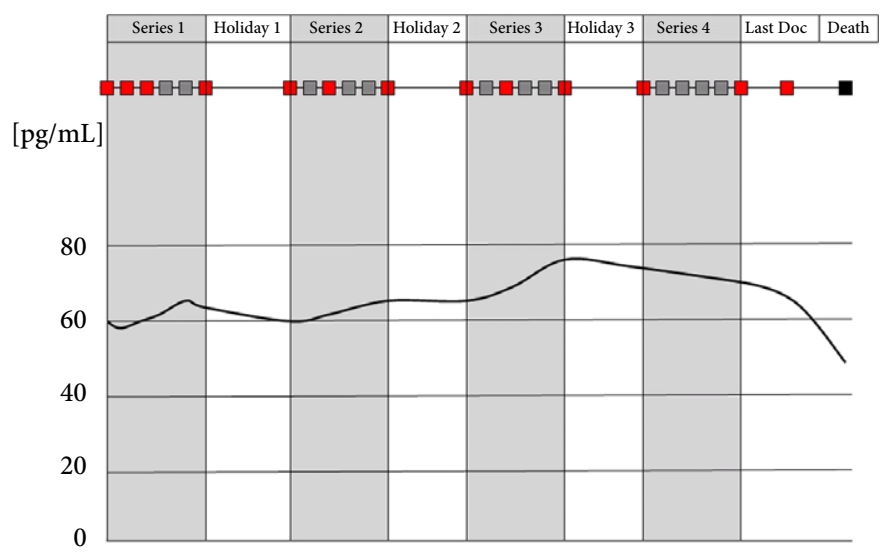

the last few years, the landscape of treatment options for CRPC has significantly changed as active, life-prolonging agents such as cabazitaxel, abiraterone-acetate and enzalutamide have become available for patients who failed docetaxel treatment. Men with docetaxel-resistant tumours, independently of having initial or acquired resistance, may benefit from the timely use of these new treatments.

Currently, a large meta-analysis confirmed the role of alkaline phosphatase and lactate dehydrogenase and newly identified serum aspartate aminotransferase as a strong predictor of survival in docetaxel-treated patients with CRPC [23]. A proteomic approach showed that increasing serum MIC-1 levels following the first cycle of docetaxel treatment are associated with poor survival [24]. Others found circulating miRNAs to be associated with docetaxel resistance [25]. Circulating biomarkers may better reflect molecular heterogeneity and hold the potential to capture tumour heterogeneity; therefore, when combining with established 
baseline clinical variables, circulating biomarkers may help to increase the accuracy of predictive models.

We found significantly lower MMP-7 levels in serum samples of men who were pretreated with estramustine phosphate and vinorelbine, suggesting that the anti-tumour effect of these agents might also have an impact on serum MMP-7 levels. Thus, MMP-7 might be used for monitoring treatment response or for the detection of developing therapy resistance. To test this hypothesis, we determined MMP-7 levels at various stages during docetaxel treatment; however, none of the changes in any of the assessed serum proteins during docetaxel treatment were associated with survival. By contrast, a PSA increase in the first and second treatment holidays, as well as an MMP-7 increase in the second holiday, were predictive for survival suggesting both serum proteins as candidates for therapy monitoring markers. In accordance, PSA levels showed characteristic changes with consequently decreasing levels during docetaxel treatment and increasing concentrations in treatment holidays. MMP-7 levels exhibited similar characteristics only when cases with high baseline levels were considered, suggesting the existence of an MMP7-expressing docetaxel-resistant molecular subtype of PCa. This might give the clinical rationale to consider MMP-7 as a target for therapy. As the initial therapeutic concept that general, systemic inhibition of a broad spectrum of MMPs might significantly improve survival has failed, current research focuses on specific inhibition of single MMPs. The first MMP-7-specific inhibitor has recently been developed [26]. Our results suggest that a specific, MMP-7-targeted therapy might improve sensitivity of PCa to docetaxel treatment; however, despite a large body of evidence in several cancers showing that MMP-7 is causally involved in chemotherapy resistance, we cannot state with certainty that this is also the case in PCa. Further functional analyses are therefore needed to determine whether the association between MMP-7 and docetaxel resistance is a coincidence or a causal relationship. Because the anti-apoptotic impact of MMP-7 was related to its proteolytic effect on sFas and FasL, we sought correlation between their serum levels. MMP-7 serum concentrations were significantly and linearly correlated with those of the extracellular domain of receptor Fas (sFas). This may underline the role of MMP-7 in the degradation of Fas and suggest an anti-apoptotic effect for MMP-7 in PCa.

In conclusion, our data identify elevated MMP-7 and sFas serum levels as markers of a docetaxel-resistant $\mathrm{PCa}$ phenotype. In addition, pretreatment MMP-7 levels were independently associated with poor patient survival, making MMP-7 a promising therapy-predicting marker in CRPC. Finally, not only the baseline levels but also the changes in PSA and MMP-7 levels in treatment holidays were predictive for survival, suggesting these serum proteins could be used as therapy-monitoring markers. These results need to be validated in larger prospective studies before being proposed for everyday clinical practice.

\section{Acknowledgements}

This work was supported by the National Research, Development and Innovation Office - NKFIH/PD 115616 and NVKP_16-1-2016-004 Grant number: FK 124431. T. Szarvas was supported by a János Bolyai Research Scholarship from the Hungarian Academy of Sciences.

\section{Conflict of Interest}

None declared.

\section{References}

1 Tannock IF, de Wit R, Berry WR et al. Docetaxel plus prednisone or mitoxantrone plus prednisone for advanced prostate cancer. $N$ Engl J Med 2004; 351: 1502-12

2 Petrylak DP, Tangen CM, Hussain MH et al. Docetaxel and estramustine compared with mitoxantrone and prednisone for advanced refractory prostate cancer. N Engl J Med 2004; 351: 1513-20

3 Cornford P, Bellmunt J, Bolla $\mathrm{M}$ et al. EAU-ESTRO-SIOG Guidelines on Prostate Cancer. Part II: treatment of Relapsing, Metastatic, and Castration-Resistant Prostate Cancer. Eur Urol 2017; 71: 630-42

4 Caffo O, Pappagallo G, Brugnara S et al. Multiple rechallenges for castration-resistant prostate cancer patients responding to first-line docetaxel: assessment of clinical outcomes and predictive factors. Urology 2012; 79: 644-9

5 Oudard S, Kramer G, Caffo O et al. Docetaxel rechallenge after an initial good response in patients with metastatic castration-resistant prostate cancer. BJU Int 2015; 115: 744-52

6 Sartor AO, Fitzpatrick JM. Urologists and oncologists: adapting to a new treatment paradigm in castration-resistant prostate cancer (CRPC). BJU Int 2012; 110: 328-35

7 Ansell A, Jerhammar F, Ceder R, Grafström R, Grénman R, Roberg K. Matrix metalloproteinase-7 and -13 expression associate to cisplatin resistance in head and neck cancer cell lines. Oral Oncol 2009; 45: 866-71

8 Liu H, Zhang T, Li X et al. Predictive value of MMP-7 expression for response to chemotherapy and survival in patients with non-small cell lung cancer. Cancer Sci 2008; 99: 2185-92

9 Mitsiades N, Yu WH, Poulaki V, Tsokos M, Stamenkovic I. Matrix metalloproteinase-7-mediated cleavage of Fas ligand protects tumour cells from chemotherapeutic drug cytotoxicity. Cancer Res 2001; 61: 577-81

10 Vargo-Gogola T, Fingleton B, Crawford HC, Matrisian LM. Matrilysin (matrix metalloproteinase-7) selects for apoptosis-resistant mammary cells in vivo. Cancer Res 2002; 62: 5559-63

11 Almendro V, Ametller E, García-Recio S et al. The role of MMP7 and its cross-talk with the FAS/FASL system during the acquisition of chemoresistance to oxaliplatin. PLOS ONE 2009; 4: e4728

12 Gallego R, Codony-Servat J, García-Albéniz X et al. Serum IGF-I, IGFBP-3, and matrix metalloproteinase-7 levels and acquired chemoresistance in advanced colorectal cancer. Endocr Relat Cancer 2009; 16: 311-7

13 Liu H, Zhang T, Wu B, Huang J, Zhou Y, Zhu J. Chronic exposure to exogenous matrilysin induces chemoresistance and enhances Bcl-2 expression in A549 lung adenocarcinoma cells. Mol Biol Rep 2009; 36: 2099-109

14 Fingleton B, Vargo-Gogola T, Crawford HC, Matrisian LM. Matrilysin [MMP-7] expression selects for cells with reduced sensitivity to apoptosis. Neoplasia 2001; 3: 459-68 
15 Szarvas T, Becker M, Vom Dorp F et al. Elevated serum matrix metalloproteinase 7 levels predict poor prognosis after radical prostatectomy. Int J Cancer 2011; 128: 1486-92

16 Bubley GJ, Carducci M, Dahut W et al. Eligibility and response guidelines for phase II clinical trials in androgen-independent prostate cancer: recommendations from the Prostate-Specific Antigen Working Group. J Clin Oncol 1999; 17: 3461-7

17 Therasse P, Arbuck SG, Eisenhauer EA et al. New guidelines to evaluate the response to treatment in solid tumours. European Organization for Research and Treatment of Cancer, National Cancer Institute of the United States, National Cancer Institute of Canada. J Natl Cancer Inst 2000; 92: 205-16

18 Harrell FE Jr. Regression Modelling Strategies with Applications to Linear Models, Logistic Regression, and Survival Analysis, New York, NY: Springer, 2001

19 Ii M, Yamamoto H, Adachi Y, Maruyama Y, Shinomura Y. Role of matrix metalloproteinase-7 (matrilysin) in human cancer invasion, apoptosis, growth, and angiogenesis. Exp Biol Med 2006; 231: 20-7

20 Knox JD, Wolf C, McDaniel K et al. Matrilysin expression in human prostate carcinoma. Mol Carcinog 1996; 15: 57-63

21 Eeles RA, Olama AA, Benlloch S et al. Identification of 23 new prostate cancer susceptibility loci using the iCOGS custom genotyping array. Nat Genet 2013; 45: 385-91

22 Lynch CC, Hikosaka A, Acuff HB et al. MMP-7 promotes prostate cancer-induced osteolysis via the solubilization of RANKL. Cancer Cell 2005; 7: 485-96

23 Guinney J, Wang T, Laajala TD et al. Prediction of overall survival for patients with metastatic castration-resistant prostate cancer: development of a prognostic model through a crowdsourced challenge with open clinical trial data. Lancet Oncol. 2017; 18: 132-42

24 Zhao L, Lee BY, Brown DA et al. Identification of candidate biomarkers of therapeutic response to docetaxel by proteomic profiling. Cancer Res 2009; 69: 7696-703

25 Lin HM, Castillo L, Mahon KL et al. Circulating microRNAs are associated with docetaxel chemotherapy outcome in castration-resistant prostate cancer. Br J Cancer 2014; 110: 2462-71

26 Edman K, Furber M, Hemsley P et al. The discovery of MMP7 inhibitors exploiting a novel selectivity trigger. Chem Med Chem 2011; 6: $769-73$
Correspondence: Tibor Szarvas PhD, Department of Urology, Semmelweis University Üllöi ut 78/b, 1082 Budapest, Hungary.

e-mail: sztibusz@gmail.com

Abbreviations: CRPC, castration-resistant prostate cancer; ECOG, Eastern Cooperative Oncology Group; FasL, fas ligand; $\mathrm{HR}$, hazard ratio; $\mathrm{HR}$, hazard ratio; MMP-7, matrix metalloproteinase 7; OR, odds ratio; $\mathrm{PCa}$, prostate cancer; RECIST, Response Evaluation Criteria in Solid Tumours; sFas, soluble Fas.

\section{Supporting Information}

Additional Supporting Information may be found online in the Supporting Information section at the end of the article:

Fig. S1. Kaplan-Meier survival plots stratified by (A) PSA level and presence of metastasis, by MMP-7 in the subgroup of (B) patients with low PSA \& and no metastases and (C) patients with high PSA and metastasis. MMP-7 is significantly associated with survival in both subgroups.

Fig. S2. Kaplan-Meier curves of overall survival according to (A) MMP-7-, (B) PSA-changes and (C) their combination during the $2^{\text {nd }}$ treatment-free period ( $2^{\text {nd }}$ holiday).

Fig. S3. Changes of PSA (blue lines) and MMP-7 levels (red lines) in representative cases of CRPC. Grey area represents treatment periods (series). Note that PSA and MMP-7 levels elevate prior or at the time of metastatic progression.

Table S1. MMP-7, sFas and FasL serum levels at baseline and at time of radiographic progression. 